\title{
Editorial: Nine deadly modelling sins
}

In the belief that knowing the most common mistakes direct marketers make might spur remedial research, I offer the following nine observations, based primarily on talking with direct marketers who have attended our DMA sponsored seminars in statistics and modelling.

- confusing response model scores with forecasts of expected response rates

- extrapolation from a biased sample

- using only one model to score a large customer database

- creating segmentation schemes that cannot be implemented across the database

- using models that contain far too many variables, ie over-fitting the data

- relying on 'automated' modelling tools and skipping the data preparation step

- not using 'zip-code' models to prune response lists

- trying to fight attrition without an attrition model

— failing to test models continuously.

\section{CONFUSING RESPONSE MODEL SCORES WITH FORECASTS OF EXPECTED RESPONSE RATES}

Most direct marketers use logistic regression to build response models. What they frequently forget is that the scores their models produce, probabilities of response, are conditioned by the overall response rate reflected in the dataset. If the same file is promoted again in a much better or a much worse month or season, the scores need to be adjusted to reflect changing conditions. Of course, if all the model is being used for is to rank a file from high to low probabilities, the adjustment does not have to be made, but if accurate response rates are required then the scores must be treated as an index to be multiplied by the new expected average response rate.

\section{EXTRAPOLATION FROM A BIASED SAMPLE}

A related problem is using a model that was built on a biased sample of the population, say an RFM model that selected the top 20 per cent of a customer file, and then applying that model to the entire file. The model will obviously not work well across the entire file.

\section{USING ONLY ONE MODEL TO SCORE A LARGE CUSTOMER DATABASE}

Large databases generally contain customers that have been on the file various lengths of time - a few months to a few years. It is unreasonable to think that the same variables that will be predictors of behaviour for new customers will predict behaviour for customers with greater tenure. For 
example, demographics may be important predictors for new customers, whereas the behaviour of older tenured customers can be predicted on the basis of their transaction data. Some, but not enough, direct marketers use CHAID to determine the right tenure breaks and to get a quick handle on the variables that are important to each tenure-defined segment.

\section{CREATING SEGMENTATION SCHEMES THAT CANNOT BE IMPLEMENTED ACROSS THE DATABASE.}

It happens all the time. Direct marketers who want to know more about their customers' attitudes toward them, the competition and the category in general, commission a large research project, a few thousand interviews/questionnaires are completed, the data go to the statisticians where factor and cluster analyses are performed and four to eight meaningful segments are produced. So far so good, but how are each of the thousands or millions of customers on the database assigned to these segments. Maybe they cannot be.

There is no guarantee that the behaviour and demographic behaviour collected on all the customers will predict segment membership, or at least predict it well enough so that it can be used. That is why it is suggested segmentation schemes be built based on behaviour and demographics and then researching each of the segments to find out what makes the members of each segment behave the way they do.

\section{USING MODELS THAT CONTAIN FAR TOO MANY VARIABLES: OVER-FITTING THE DATA}

Direct marketers who are not statisticians still behave as if they believe that bigger is better, and that may be true, in certain situations, but when it comes to modelling the conventional wisdom may not hold. Models with large numbers of variables, more than eight to ten, and especially models in which the variables differ markedly in terms of their significance ( $t$ values and chi-square scores) will frequently over-fit the modelling dataset and will not work well when rolled out. The simple, but not always followed, practice of eliminating variables with relatively low significance scores, and checking to see if the validation runs (decile analyses) have changed can solve this potential problem.

\section{RELYING ON 'AUTOMATED' MODELLING TOOLS AND SKIPPING THE DATA PREPARATION STEP}

What more can be said? Automated modelling tools do not do the best job possible. But a trained analyst, using the same tools and a little care - correcting for non-linear relationships, identifying interaction variables - creating new variables from combinations of old ones, using principal component analysis, instead of large numbers of dummy variables, will produce a much better model. While modelling may break the 'bigger is better rule', the 'there's no free lunch' rule still holds

\section{NOT USING 'ZIP-CODE' MODELS TO PRUNE DOWN RESPONSE LISTS}

Finally, there is no good reason why large mailers cannot make marginal lists work with the aid of simple zip-plus four census data models. These models will generally let the marketer find 10 per cent to 20 per cent of a marginal list that will work for them. And, it is self-funding, in that the same model will let the marker find 10 per cent to 20 per cent of a list that is working on average, 
but would work even better if the bottom scored portion of the file were not promoted.

\section{TRYING TO FIGHT ATTRITION WITHOUT AN ATTRITION MODEL}

Trying to reduce attrition rates without targeting likely attriters is economically unfeasible. Even if the attrition rate is as high as 10 per cent - 90 per cent are still staying. The 10 per cent have to be targeted, and it is not hard to do. A model may not even be required if the bulk of the attrition takes place in the first few months of the customer relationship, in which case what is needed is good new customer care.

\section{TRYING TO FIGHT ATTRITION WITHOUT AN ATTRITION MODEL}

Promoting to only the top deciles is a huge mistake. Without mailing or calling into the bottom deciles there is no way of knowing if the model is working or if there has been a processing or a scoring error. Just looking at the response rates will not tell anything because conditions may have changed ('confusing response model scores' \#1 above). Mailing a random sample is alright, but I prefer sampling and coding each decile.

DAVID SHEPARD Editorial Board May 2001 